American Journal of Applied Sciences 5 (4): 435-439, 2008

ISSN 1546-9239

(C) 2008 Science Publications

\title{
Electronic Energies in Delta Doped AIGaAs/GaAs Heterostuctures
}

\author{
${ }^{1}$ A. Meftah, ${ }^{1}$ H. Ajlani, ${ }^{1}$ A. Marzouki, ${ }^{2}$ R. Chtourou and ${ }^{1}$ M. Oueslati \\ ${ }^{1}$ Faculty of Sciences of Tunis, University campus, 2092 El Manar, Tunisia \\ ${ }^{2}$ National Institute of Scientific and Technical Research
}

\begin{abstract}
The band bending of the potential at the $\mathrm{AlGaAs} / \mathrm{GaAs}$ interface increases with the electrons concentration in the GaAs channel. This band bending is often ignored in the theoretical calculation of the electronic energies and a linear potential approximation was used. This led to imprecise energy values and occupied subband configurations. We have taken account of the band bending in the subband energies calculation. The potential expression was considered as sum of linear and quadratic terms. The quadratic term was considered as a time independent perturbation and the electronic energies were corrected to the first order. We have calculated the Fermi energy for different electronic concentrations. The relation between the Fermi energy and the electronic concentration at the interface leads to the desired configuration of the occupied subbands. When the fundamental subband is only occupied, the characteristics of those heterostructures are best enhanced. The corresponding electronic concentration was calculated. The theoretical results were used for the determination of the active electron concentration in two $\mathrm{AlGaAs} / \mathrm{GaAs}$ heterostructures.
\end{abstract}

Keywords: $\mathrm{AlGaAs} / \mathrm{GaAs}$ interface, electronic energies.

\section{INTRODUCTION}

In $\mathrm{AlGaAs} / \mathrm{GaAs}$ heterostructures, a two dimensional electron gas (2DEG) is formed in the GaAs channel near the $\mathrm{AlGaAs} / \mathrm{GaAs}$ interface ${ }^{[1]}$. In the plane of the 2DEG, electrons have high mobility and high concentration reaching $10^{13} \mathrm{~cm}^{-2]}$. Those electron characteristics are used in the fabrication of high speed optoelectronic devices. More sophistically device engineering requires an accurate knowledge of subbands structure and Fermi's level localisation.

In $\mathrm{AlGaAs} / \mathrm{GaAs}$ heterostructures, electronic structures are often numerically calculated using self consistent resolution of Schrödinger and Poisson equations $^{[3-8]}$ or using SILVACO Mercury software. In theoretical band structure calculation, the GaAs conduction band bending near the $\mathrm{AlGaAs} / \mathrm{GaAs}$ interface is often ignored, and a triangular potential is considered ${ }^{[9]}$. Schubert and all have approximated the GaAs band edge by a polygonal curve ${ }^{[10]}$ and the electron energy is calculated by matching the electron De Broglie wave light and the width of the triangular well.

In this paper, we show that the triangular potential introduced in the current density equation gives rise to a spatial dependence of the electronic concentration which can be approximated by a linear function depending of the distance $\mathrm{z}$ to the AlGaAs/GaAs interface. The resolution of the Poisson equation gives rise to an approximated potential $V(z)=a z-b z^{2}$. The linear term is first considered in the Schrödinger equation and the electronic energies and wave functions are calculated in the basic of the effective mass approximation. The quadratic term is treated as a time independent perturbation and the electronic energies are corrected to the first order. The configurations of occupied subbands are related to the Fermi energy. This energy is calculated for different concentration of the two dimensional electron gas.

Electrical field and potential expressions near the AlGaAs/GaAs interface: Near the AlGaAs/GaAs interface, a linear potential $V(z)=a z$ is often considered. By writing the current-density and the Poisson equations, we will carry the electrical field and potential expressions.

The current-density equation for electrons in the GaAs channel is:

$e n_{2 D E G} \mu_{e} \vec{E}+e D_{e} \vec{\nabla} n_{2 D E G}=\overrightarrow{0}$

$\vec{E}$ is the electrical field. $n_{2 D E G}, e, \mu_{e}$ and $D_{e}$ are electron concentration, charge, mobility and drift coefficient respectively.

The resolution of equation (1) gives:

Corresponding Author: Abdelaziz MEFTAH, Faculty of Sciences of Tunis, University campus, 2092 El Manar, Tunisia 
$n_{2 D E G}(z)=n_{2 D E G}(0) e^{-\frac{\mu_{e} a}{e D_{e}} z}$

For weak value of $z$, one can write:

$n_{2 D E G}(z)=-\alpha\left(z-z_{0}\right)$

Where $\alpha=\frac{\mu_{e} a}{e D_{e}} n_{2 D E G}(0)$ and $z_{0}=\frac{e D_{e}}{\mu_{e} a}$ is the

flat band beginning abscise.

In the two dimensional electron gas approximation, all electrons are supposed to be in the interface plane where the electron concentration is $n_{2 D E G}$. We can write:

$n_{2 D E G}=\int_{0}^{z_{0}} n_{2 D E G}(z) d z$

This gives: $\alpha=\frac{2 n_{2 D E G}}{z_{0}^{2}}$

The resolution of the Poisson equation gives:

$E(z)=\frac{e \alpha}{\varepsilon}\left(\frac{z_{0}^{2}}{2}+\frac{z^{2}}{2}-z z_{0}\right)$

and $V(z)=\frac{e^{2} \alpha}{\varepsilon}\left(\frac{z_{0}^{2}}{2} z-\frac{z_{0}}{2} z^{2}+\frac{z^{3}}{6}\right)$

Where $\varepsilon$ is the GaAs dielectric-permittivity constant

To the first order approximation we have

$V(z)=\frac{e^{2} \alpha z_{0}^{2}}{2 \varepsilon} z=\frac{e^{2} n_{2 D E G}}{\varepsilon} z=a z$,

then

$a=\frac{e^{2} n_{2 D E G}}{\varepsilon}$.

To the second order approximation, the potential is:

$V(z)=a z-b z^{2}$

$V(z)$ is constant for $z \geq z_{0}$, then $b=\frac{a}{2 z_{0}}$

Subband energies in the triangular potential: In the basic of the effective mass approximation, the Schrödinger equation in the GaAs channel is:

$$
\begin{aligned}
& \left(\frac{P_{/ /}^{2}}{2 m_{/ /}^{*}}+\frac{P_{\perp}^{2}}{2 m_{\perp}^{*}}+a z\right) \psi(\vec{r})= \\
& \left(E-E_{g}^{G a A s}\right) \psi(\vec{r})
\end{aligned}
$$

$P_{/ /}, m_{/ /}^{*}$ and $P_{\perp}, m_{\perp}^{*}$ are electron momentum and effective masse in the directions parallel and perpendicular to the interface respectively. $E_{g}^{G a A s}$ is the GaAs band gap.

In literature, no difference between the effective masses $m_{/ /}^{*}$ and $m_{\perp}^{*}$ were reported, we then write:

$$
m_{/ /}^{*}=m_{\perp}^{*}=m^{*}
$$

The calculus of the wave functions and energies are given in the appendix. The wave function is $\psi(\vec{r})=$ $\frac{1}{\sqrt{S}} e^{i \vec{k}_{/ /} \vec{r}_{/ /}} \varphi(z)$, where $\vec{k}_{/ /}$and $\vec{r}_{/ /}$are the wave and position vectors parallel to the interface respectively.

$\varphi(z)$ is the Airy function $A_{i}\left(\left(\frac{2 m^{*} a}{\hbar^{2}}\right)^{\frac{1}{3}}\left(z-\frac{\varepsilon_{i}}{a}\right)\right)$ and the energy is $E_{i}=\varepsilon_{i}+E_{g}^{G a A s}+\frac{\hbar^{2} k_{/ /}^{2}}{2 m^{*}}$ with

$$
\varepsilon_{i}=\left(\frac{3 \pi}{2}\right)^{\frac{2}{3}}\left(\frac{\hbar e^{2} n_{2 D E G}}{\sqrt{2 m^{*}} \varepsilon}\right)^{\frac{2}{3}}(i+0.75)^{\frac{2}{3}}
$$

Where $i=0,1,2,3 \ldots$

For $m^{*}=0.067 * m$ and $\varepsilon=1301 * 8.85 * 10^{-12} S I$, the energy $\varepsilon_{i}(\mathrm{meV})$ is given by:

$$
\varepsilon_{i}(m e V)=0.997 * 10^{-6} n_{2 D E G}^{\frac{2}{3}}(i+0.75)^{\frac{2}{3}}
$$

Where $n_{2 D E G}$ is expressed in $\mathrm{cm}^{-2}$

We mismatch the subband energies calculated by the expression (4) and those found in literature, for the 2DEG concentration $n_{2 D E G}=5.10^{11} \mathrm{~cm}^{-2}$. The energies $\varepsilon_{0}$ and $\varepsilon_{1}$ of the fundamental and the first excited levels respectively are given in table 1 .

Energy correction:-The first order correction of the energy $\varepsilon_{i}$ is: 
Table 1: Energies $\varepsilon_{0}$ and $\varepsilon_{1}$ of the fundamental and first excited levels in the triangular potential of the AlGaAs/GaAs heterostructure. The first row shows energies given by this work. The other rows show energies found in literature.

\begin{tabular}{lccccccc}
\hline $\begin{array}{c}\text { References } \\
\text { Energies }\end{array}$ & This work & {$[3]$} & {$[9]$} & {$[10]$} & {$[11]$} & {$[12,13]$} & {$[14]$} \\
\hline$\varepsilon_{0}(\mathrm{meV})$ & 51.8 & 45.7 & 69.8 & 64.5 & 50 & 67.9 & 73.1 \\
$\varepsilon_{1}(\mathrm{meV})$ & 91.2 & 71.9 & 122.7 & 76.0 & 73 & 93.2 & 93.2 \\
\hline
\end{tabular}

Table 2: $\alpha$ and $\beta$ values, and fundamental and 5 first excited levels energies before and after corrections for

\begin{tabular}{ccccccc}
\multicolumn{7}{c}{$n_{2 D E G}=5^{*} 10^{11} \mathrm{~cm}^{-2}$ and $z_{0}=500 . \AA$} \\
\hline$i$ & 0 & 1 & 2 & 3 & 4 & 5 \\
\hline$\alpha$ & -0.779 & -1.363 & -1.840 & -2.262 & -2.648 & -3.008 \\
$\beta$ & 1.093 & 3.342 & 6.095 & 9.212 & 12.622 & 16.282 \\
$\varepsilon_{i}(\mathrm{meV})$ & 51.8 & 91.2 & 123.3 & 151.6 & 177.5 & 201.6 \\
$\left(\varepsilon_{i}+\varepsilon_{i}^{(1)}\right)(\mathrm{meV})$ & 49.3 & 83.2 & 108.7 & 129.6 & 147.3 & 162.6
\end{tabular}

$\varepsilon_{i}^{(1)}=\frac{\left\langle\varphi_{i}(Z)\left|-b z^{2}\right| \varphi_{i}(Z)\right\rangle}{\left\langle\varphi_{i}(Z) \mid \varphi_{i}(Z)\right\rangle}$

Where $Z=\left(\frac{2 m^{*} a}{\hbar^{2}}\right)^{\frac{1}{3}}\left(z-\frac{\varepsilon}{a}\right)$

$\varepsilon_{i}^{(1)}=-\frac{a}{2 z_{0}}\left\{\frac{\varepsilon_{i}^{2}}{a^{2}}+2 \alpha \frac{\varepsilon_{i}}{a}\left(\frac{\hbar^{2}}{2 m^{*} a}\right)^{\frac{1}{3}}+\right.$ $\beta\left(\frac{\hbar^{2}}{2 m^{*} a}\right)^{\frac{2}{3}}$

Where $\alpha=\frac{I_{1 i}}{I_{0 i}}, \beta=\frac{I_{2 i}}{I_{0 i}}, I_{0 i}=\int_{r_{i}}^{\infty} A_{i}^{2}(Z) d Z$,

$I_{1 i}=\int_{r_{i}}^{\infty} Z A_{i}^{2}(Z) d Z$ and $I_{2 i}=\int_{r_{i}}^{\infty} Z^{2} A_{i}^{2}(Z) d Z$

$I_{0 i}, I_{1 i}$ et $I_{2 i}$ are calculated by computer. $\varepsilon_{i}^{(1)}$ is related to $\varepsilon_{i}, n_{2 D E G}$ and $z_{0}$ by:

$$
\varepsilon_{i}^{(1)}=-\frac{1}{z_{0}}\left\{0.362 * 10^{12} \frac{\varepsilon_{i}^{2}}{n_{2 D E G}}+\right.
$$

$$
1.602 * 10^{5} \alpha \varepsilon_{i} n_{2}^{-\frac{1}{D}}
$$$$
\left.1.773 * 10^{-2} \beta n_{2 D E G}^{\frac{1}{3}}\right\}
$$

$z_{0}, n_{2 D E G}$ and $\varepsilon_{i}$ are expressed in $\mathrm{A}, \mathrm{cm}^{-2}$ and meV respectively.

For $n_{2 D E G}=5 * 10^{11} \mathrm{~cm}^{-2}$ and $z_{0}=500 \mathrm{~A}$ the $\alpha$ and $\beta$ coefficients and the corrected energies for the fundamental and the five first excited levels are given in table 2.

The evolutions with $n_{2 D E G}$ of the energies $\varepsilon_{i}$ and $\varepsilon_{i}+\varepsilon_{i}^{(1)}$ for $i=0,1$ and 2 are represented in figure 1. For each level, the energy correction is negative, its absolute value increases with increasing $n_{2 D E G}$. 


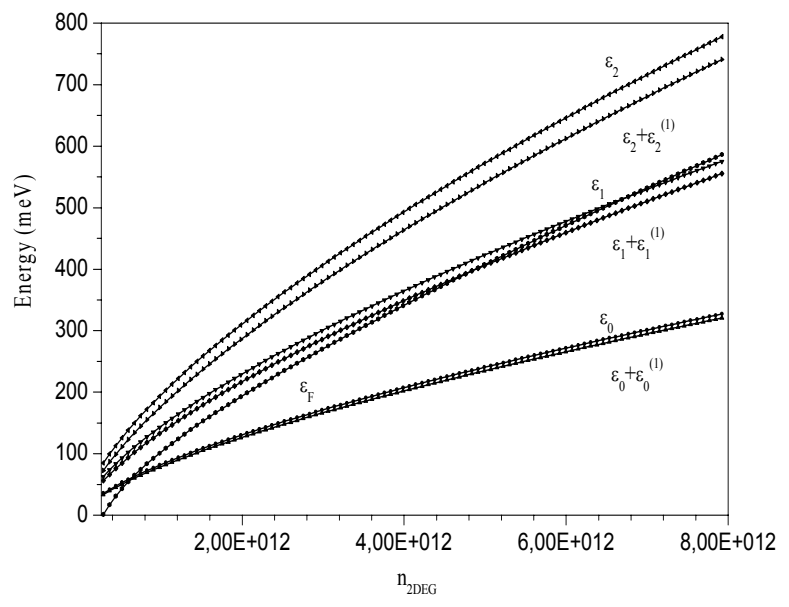

Fig. 1: Evolution with $n_{2 D E G}$ of the fundamental, two first excited and Fermi levels energies before and after the correction.

The evolutions with the well width $z_{0}$ of the energy correction for the three levels are represented in figure 2. For weak $z_{0}$ value, the band bending is more pronounced near the $\mathrm{AlGaAs} / \mathrm{GaAs}$ interface, the linear potential approximation is insufficient and the energy correction for each level is important. For large $z_{0}$ value, the potential is rigorously linear near the $\mathrm{AlGaAs} / \mathrm{GaAs}$ interface and the energy correction is weak.

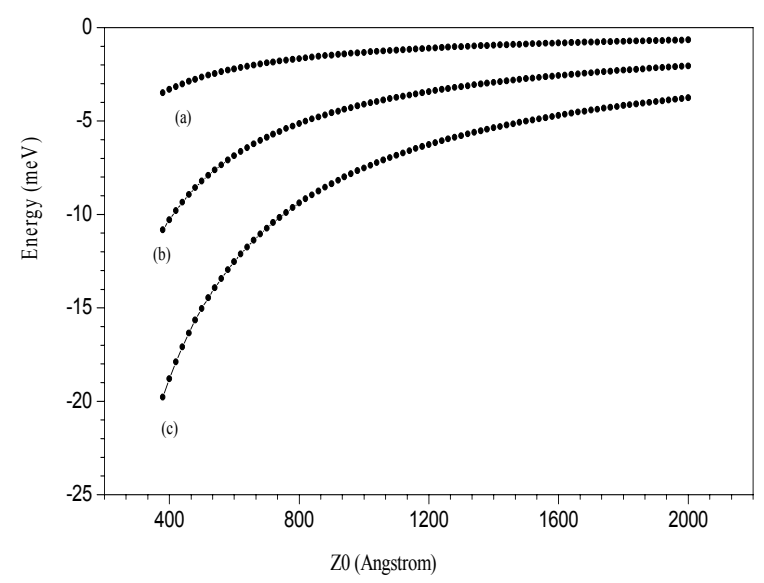

Fig. 2: Evolution with the potential-well width of the energy corrections of the fundamental (a), the first (b) and second (c) excited levels

The Fermi energy: The over all electron concentration in the two dimensional electron gas is given by ${ }^{[15]}$ : $n_{2 D E G}=\frac{m_{e}}{\pi \hbar^{2}} K T \sum_{i} \log \left(1+e^{\frac{\varepsilon_{F}-\varepsilon_{i}}{K T}}\right)$

Where $\mathrm{T}$ and $\mathrm{K}$ are temperature and Boltzman constant. $n_{2 D E G}$ could be written:

$n_{2 D E G}$

$=\frac{m_{e}}{\pi \hbar^{2}} K T \log \left(\pi\left(1+e^{\frac{\varepsilon_{F}-\varepsilon_{i}}{K T}}\right)\right)=\frac{m_{e}}{\pi^{2}} K T \log A$

Where

$A=\left(1+a e^{-\frac{\varepsilon_{0}}{K T}}\right)\left(1+a e^{-\frac{\varepsilon_{1}}{K T}}\right) \ldots\left(1+a e^{-\frac{\varepsilon_{N}}{K T}}\right)$

With $a=e^{\frac{\varepsilon_{F}}{K T}}$ and $\varepsilon_{N}$ is the energy of the upper confined level

The condition $\varepsilon_{i}>K T$ is often satisfied, thus $e^{-\frac{\varepsilon_{i}}{K T}}$ is weak and we can approximate $A$ to the second order in $a$ and write:

$$
A=1+a \sum_{i=0}^{N} e^{-\frac{\varepsilon_{i}}{K T}}+a^{2}\left(e^{-\frac{\varepsilon_{0}}{K T}} \sum_{i=1}^{N} e^{-\frac{\varepsilon_{i}}{K T}}+e^{-\frac{\varepsilon_{1}}{K T}} \sum_{i=2}^{N} e^{-\frac{\varepsilon_{i}}{K T}}+\ldots+e^{-\frac{\varepsilon_{N-1}}{K T}} e^{-\frac{\varepsilon_{N}}{K T}}\right)
$$

We put:

$$
\begin{aligned}
& \alpha=\sum_{i=0}^{N} e^{-\frac{\varepsilon_{i}}{K T}} \text { and } \\
& \beta=\sum_{j=0}^{N-1} e^{-\frac{\varepsilon_{j}}{K T}}\left(\sum_{i=j+1}^{N} e^{-\frac{\varepsilon_{i}}{K T}}\right)
\end{aligned}
$$

The equations (8) and (9) give:

$$
1+\alpha a+\beta a^{2}=A=e^{\frac{\pi \hbar^{2}}{m_{e} K T} n_{2 D E G}}
$$

The resolution of (10) gives:

$$
\left.\varepsilon_{F}=K T L 0 \% \frac{-\sum_{i=0}^{N} e^{-\frac{\varepsilon_{i}}{K T}}+\sqrt{\left(\sum_{i=0}^{N} e^{-\frac{\varepsilon_{i}}{K T}}\right)^{2}+4\left(e^{\frac{\pi \hbar^{2}}{m_{e} K T} n_{2 D E G}}-1\right) \sum_{j=0}^{N-1} e^{-\frac{\varepsilon_{j}}{K T}}\left(\sum_{i=j+1}^{N} e^{-\frac{\varepsilon_{i}}{K T}}\right)}}{2 \sum_{j=0}^{N-1} e^{-\frac{\varepsilon_{j}}{K T}}\left(\sum_{i=j+1}^{N} e^{-\frac{\varepsilon_{i}}{K T}}\right)}\right\}
$$

The Fermi energy $\varepsilon_{F}$ is calculated by computer. The evolution of $\varepsilon_{F}$ with $n_{2 D E G}$ is shown in figure 1.

The fundamental level is populated for 
$n_{2 D E G}>6.3 * 10^{11} \mathrm{~cm}^{-2}$. The first excited level is

populated for $n_{2 D E G}>4.8 * 10^{12} \mathrm{~cm}^{-2}$. The

$n_{2 D E G}$ can be related to the doping concentration, which choice leads to the desired configuration of the occupied subbands.

\section{CONCLUSION}

In GaAs channel and near the $\mathrm{AlGaAs} / \mathrm{GaAs}$ interface, the potential is approximated by the expression $V(z)=a z-b z^{2}$. The quadratic term is weak compared to the linear one. To first approximation the potential is triangular. The subband energies and wave functions are exactly calculated in the basic of the effective mass approximation. The quadratic term is considered as time independent perturbation, and energies are corrected to the first order. The calculated subband energies agree well with that found in literature.

The Fermi energy is calculated for different concentrations $n_{2 D E G}$ of the two dimensional electron gas. The concentration $n_{2 D E G}$ can be related to doping concentration, thus the desired configuration of the occupied subbands will be obtained by the choice of the doping concentration.

\section{REFERENCES}

1. Störmer, L. H., R. Dingle, A. C. Gossard, W. Wiegman and M. D. Sturge, 1983. TWO DIMENSIONA L ELECTRON GAS AT SEMICONDUCTOR-SEMICONDUCTOR INTERFACE. Solid. Stat. Com., 29: 705-709.

2. Shubert, E. F., J. E. Cunnigham and W. T. Tsang, 1987. Electron-Mobility Enhancement and Electron concentration Enhancement in $\delta$-doped nGaAs at 300K. Solid. Stat. Com., 63: 591-594.

3. Stern, F.and S. Das Sarma, 1984. Electron energy levels in GaAs- $\mathrm{Ga}_{1-\mathrm{x}} \mathrm{Al}_{\mathrm{x}} \mathrm{As}$ heterojunctions. Phys. Rev. B, 30: 840-848.
4. Tan, I. H., G. L Snider, L. D. Chang, and E. L Hu, 1990. A self-consistent solution of SchrödingerPoisson equations using a nonuniform mesh. J. App. Phys., 68 (8): 4071-4076.

5. Bouzaiene, L., L. Sfaxi, H. Sghaier.and H. Maaref, 1999., Improvement of the electron density in the channel of an $\mathrm{AlGaAs} / \mathrm{GaAs}$ heterojunction by introducing $\mathrm{Si}$ doping in the quantum well. J. Appl. Phys., 85 (12): 8223-8227.

6. Aloulou, S., H. Ajlani, A. Meftah, M. Oueslati, L. Sfaxi and H. Maaref, 2002. Electron confinement in planar doped heterostructure $\mathrm{Ga}_{1}$. ${ }_{x} \mathrm{Al}_{\mathrm{x}} \mathrm{As}: \mathrm{Si} / \mathrm{GaAs}$. Material Sciences and engineering B, 96: 14-18.

7. Singh, R., C. M. Snowder, 1999. A charge control HEMT model incorporating deep level effects,.Solid. Stat.. Electronics, 43: 473-480.

8. Ajlani, H., A. Meftah, R. Chtourou, M. Oueslati and H. Maaref, 2006. Photoluminescence studies of confined states in $\mathrm{AlGaAs} / \mathrm{GaAs}$ asymmetric quantum well. Physica E, 33: 325-330.

9. Ando, T., A. B. Fowler and F. Stern, 1982. Electronic proprieties of two-dimensional systems. Rev. Mod. Phys., 54 (2): 437-672.

10. Schubert, E. F., 1985. Electron Subband Structure in Selectively Doped $\mathrm{n}-\mathrm{Al}_{\mathrm{x}} \mathrm{Ga}_{1-\mathrm{x}} \mathrm{As} / \mathrm{GaAs}$ Heterostructures. IEEE Trans. Electron Devices ED-32, 9: 1868-1873.

11. Ando, T., 1982. Self-Consistent Results for a $\mathrm{GaAs} / \mathrm{Al}_{\mathrm{x}} \mathrm{Ga}_{1-\mathrm{x}} \mathrm{As}$ Heterojunction Subband Structure and Light-Scattering Spectra. J. Phys. Soc. Japan, 51: 3893-3899.

12. Stern, F., 1983. Doping consideration for heterojunctions. Appl. Phys. Lett., 43: 974-976.

13. Delagebeaudeuf, D. and N. T. Lihn, 1982. Metal(n) AlGaAs-GaAs two-dimensional electron gas FET. IEEE Trans. Electron Device, ED-29: 955960.

14. Mathieu, H., 1998. Physique des semiconducteurs et des composants électroniques. Edition MASSON, Paris, Quatrième édition, pp:522. 